Article

\title{
Associations of B Vitamin-Related Dietary Pattern during Pregnancy with Birth Outcomes: A Population-Based Study in Northwest China
}

\author{
Shanshan Li ${ }^{1,+}(\mathbb{D})$, Danmeng Liu ${ }^{2,+}$, Yijun Kang ${ }^{2}$, Pengfei Qu ${ }^{3}$, Baibing Mi ${ }^{2}\left(\mathbb{D}\right.$, Zhonghai Zhu ${ }^{2}$, Lixin Han ${ }^{4,5}$, \\ Yaling Zhao ${ }^{2}$, Fangyao Chen ${ }^{2} \mathbb{D}$, Leilei Pei ${ }^{2}$, Lingxia Zeng ${ }^{2}$, Duolao Wang ${ }^{6} \mathbb{D}$, Hong Yan ${ }^{2,7}$ \\ and Shaonong Dang ${ }^{2, *(\mathbb{D})}$
}

check for updates

Citation: Li, S.; Liu, D.; Kang, Y.; Qu, P.; Mi, B.; Zhu, Z.; Han, L.; Zhao, Y.; Chen, F.; Pei, L.; et al. Associations of B Vitamin-Related Dietary Pattern during Pregnancy with Birth Outcomes: A Population-Based Study in Northwest China. Nutrients 2022, 14, 600. https://doi.org/ $10.3390 /$ nu14030600

Academic Editor: Yunxian Yu

Received: 27 December 2021

Accepted: 27 January 2022

Published: 29 January 2022

Publisher's Note: MDPI stays neutral with regard to jurisdictional claims in published maps and institutional affiliations.

Copyright: (c) 2022 by the authors Licensee MDPI, Basel, Switzerland. This article is an open access article distributed under the terms and conditions of the Creative Commons Attribution (CC BY) license (https:// creativecommons.org/licenses/by/ $4.0 /)$.
1 School of Public Health, Shandong First Medical University \& Shandong Academy of Medical Sciences, Taian 271016, China; lishanshan@sdfmu.edu.cn

2 Department of Epidemiology and Health Statistics, Health Science Center, Xi'an Jiaotong University, Xi'an 710061, China; liudanmeng1214@stu.xjtu.edu.cn (D.L.); tjkyj@xjtu.edu.cn (Y.K.); xjtu.mi@xjtu.edu.cn (B.M.); zhonghai_zhu@xjtu.edu.cn (Z.Z.); zhaoyl666@xjtu.edu.cn (Y.Z.); chenfy@xjtu.edu.cn (F.C.); peileilei424@163.com (L.P.); tjzlx@xjtu.edu.cn (L.Z.); yanhonge@xjtu.edu.cn (H.Y.)

3 Translational Medicine Center, Northwest Women's and Children's Hospital, Xi'an 710061, China; xinxi3057@stu.xjtu.edu.cn

4 Key Laboratory of Trace Elements and Endemic Diseases of National Health Commission, Health Science Center, Xi'an Jiaotong University, Xi'an 710061, China; weijianweijikongchu@shaanxi.gov.cn

5 Disease Control and Prevention Division, Shaanxi Provincial Health Commission, Xi' an 710000, China

6 Department of Clinical Sciences, Liverpool School of Tropical Medicine, Liverpool L3 5QA, UK; duolao.wang@lstmed.ac.uk

7 Nutrition and Food Safety Engineering Research Center of Shaanxi Province, Xi'an 710061, China

* Correspondence: tjdshn@xjtu.edu.cn

+ These authors contributed equally to this work.

\begin{abstract}
This study aimed to derive a maternal dietary pattern to explain the variation in B vitamins during pregnancy and to investigate this pattern in relation to birth outcomes. A total of 7347 women who gave birth to live newborns less than one year were included. Their dietary pattern during pregnancy was derived using the reduced-rank regression method with six B vitamins as response variables. Associations between dietary pattern score and birth weight, gestational age at delivery, birth weight Z score, low birth weight, preterm, and small-for-gestational-age (SGA) were estimated using generalised linear mixed models. We identified a high B-vitamin dietary pattern characterised by high intakes of animal foods, vegetables, fungi and algae, legumes, and low intakes of oils and cereals. Women in the highest quartile of this pattern score had newborns with a $44.5 \mathrm{~g}$ (95\% CI: 13.8, $75.2 \mathrm{~g}$ ) higher birth weight, 0.101 (95\% CI: 0.029, 0.172) higher birth weight Z score, and 27.2\% (OR: 0.728 ; $95 \%$ CI: $0.582,0.910$ ) lower risk of SGA than those in the lowest quartile. Our study suggested that adherence to the high B-vitamin dietary pattern during pregnancy was associated with a higher birth weight and a lower risk of SGA.
\end{abstract}

Keywords: dietary pattern; B vitamins; pregnancy; birth weight; small-for-gestational-age; reduced rank regression

\section{Introduction}

Birth outcomes, which commonly refer to birth weight and gestational age, are not only closely related to the morbidity and mortality of infants and young children [1,2], but are also key predictors of chronic non-communicable diseases in adulthood [3-5]. As the economy grows by leaps and bounds, the health status of women and children in China has been greatly improved in the past few decades [6]. However, China has the largest population in the world, of more than 1.4 billion. According to the latest WHO data, China ranks among the top five countries worldwide in terms of the number of adverse 
birth outcomes such as preterm, low birth weight (LBW), and small-for-gestational-age (SGA) [7-9].

The aetiology of most types of adverse birth outcomes is complex. As a modifiable risk factor, maternal nutrition during pregnancy has been widely discussed for its potential to prevent adverse birth outcomes [10]. B vitamins are a class of water-soluble micronutrients that act as co-enzymes in numerous catabolic and anabolic enzymatic reactions [11]. To our knowledge, the associations of other B vitamins than folate (vitamin B9) with adverse birth outcomes remain unclear. Some epidemiologic studies have reported that maternal dietary intakes of B vitamins are inversely related to the risk of adverse birth outcomes [12-14]. Since B vitamins share similarities in food sources and biological functions, these studies failed to clarify the individual effect of specific B vitamins, nor did they capture the cumulative effect of multiple B vitamins [15].

Recent nutritional epidemiological studies have shifted the focus from single nutrients to dietary patterns, which describe the overall diet and better reflect the interactive or synergistic effects of different nutrients [16]. Of note, Hoffmann et al. proposed the reduced rank regression (RRR) method, by which dietary patterns are derived using a posteriori statistical analysis, but response variables (e.g., nutrients) are chosen using a priori knowledge that suggests these variables are hypothesized to be related to health outcomes [17]. The RRR method identifies the linear combination of food groups that account for as much variation as possible in a set of response variables. Compared to traditional methods (e.g., Health Eating Index and principal component analysis), the RRR method has the advantage of building biological pathways through which diet affects health outcomes [18].

Therefore, based on the cross-sectional data in northwest China, we aimed to identify a maternal dietary pattern that maximally explains the variation in B vitamins using the RRR method. Furthermore, we sought to assess the associations of this pattern with birth outcomes after controlling for covariates.

\section{Materials and Methods}

\subsection{Study Design and Participants}

The present study used data from a cross-sectional study conducted in Shaanxi Province of northwest China. The design and methodology of the cross-sectional study have been described previously in detail $[19,20]$. Briefly, 30,027 women who were pregnant during 2010-2013 and had pregnancy outcomes were selected using the stratified multistage random sampling method. First, twenty counties and ten districts were randomly selected from the Shaanxi Province according to the proportion of urban to rural population, population density and fertility rate. Second, six townships and three streets were randomly selected from the sampled counties and districts, separately. Third, six villages and communities were randomly selected from the sampled townships and streets, separately. Finally, thirty and sixty women were randomly selected from the sampled villages and communities, separately. All participants were interviewed from August to November 2013. Information on maternal general and pregnancy characteristics, and neonatal outcomes were collected by a standardised and structured questionnaire. Furthermore, 7750 women who gave birth to live newborns less than one year ago were interviewed to obtain information on dietary intakes during pregnancy. For this study, 403 women were excluded due to multiple births $(n=87)$ or implausible energy intake $(<4500$ or $>20000 \mathrm{~kJ} / \mathrm{d})(n=316)$ [21]. Consequently, a total of 7347 women were included in the final analysis, with a median of 3 months (10-90th percentiles: 0-7 months) after delivery. A flow chart of the selection of study participants is displayed in Figure S1.

\subsection{Dietary Assessment}

The dietary intakes during pregnancy were estimated retrospectively using a semiquantitative food frequency questionnaire (FFQ). Since dietary intakes did not change to a great extent throughout pregnancy [22-24], and it was cumbersome to collect dietary data 
for different trimesters, we assessed the average dietary intakes over the whole pregnancy at one time. The FFQ applied in this study was established based on a validated FFQ designed for pregnant women during the third trimester in northwest China [25]. The validation study showed good correlations between nutrients estimated by the FFQ and six repeated $24 \mathrm{~h}$ recalls. Pearson's correlation coefficients ranged from 0.53 for cholesterol and carotene, to 0.70 for vitamin E and potassium [25]. The FFQ used in this study consisted of 107 items. For the five food items concerning edible oil and condiments, the weight consumed per month and the number of family members were recorded. For the other 102 food items, consumption frequencies were assessed on an eight-level scale (never or almost not, less than one time/month, one to three times/month, one time/week, two to four times/week, five to six times/week, one time/day, or more than two times/day), and portion sizes were estimated on a three-level scale (large, medium, or small) using the food photographs [26]. The daily intakes of total energy and nutrients were estimated by multiplying consumption frequency by the portion size of each food item and their corresponding nutrient content abstracted from the China Food Composition Table [27,28]. All nutrient intakes were adjusted for total energy intake with the use of the residual method [29].

\subsection{Birth Outcomes}

Neonatal outcomes, including sex, gestational age at birth, and birth weight were obtained from the Medical Certificate of Birth. Birth weight was measured to the nearest $10 \mathrm{~g}$. Gestational age at birth was the number of weeks from the first day of the last menstrual period to the date of delivery. The sex- and gestational age-adjusted birth weight Z score was calculated based on the International Fetal and Newborn Growth Consortium for the 21st Century (INTERGROWTH-21st) standards [30]. LBW referred to a birth weight less than $2500 \mathrm{~g}$ [31]. Preterm was defined as gestational age at birth less than 37 weeks [32]. SGA referred to a birth weight $Z$ score below the 10th percentile [33].

\subsection{Covariates}

The covariates selected based on previous studies can be categorised as maternal socio-demographic characteristics and health-related behaviours during pregnancy [34-36]. Socio-demographic characteristics comprised geographic area, residence, age at delivery, education, occupation, household wealth index, and parity. The principal component analysis was used to construct the household wealth index based on household income and expenditure, type of house, number of appliances, and number of vehicles [37]. Poor was defined as the first principal component below the 33.3rd percentile. Health-related behaviours included smoking, alcohol consumption, pregnancy complications, medication use, as well as folic acid, iron, calcium, and multivitamin supplementation. Only passive smoking was considered as a covariate because of pregnant women's low prevalence of active smoking in our study areas. Passive smoking was defined as a non-smoker being exposed to tobacco smoke for at least $15 \mathrm{~min}$ per day. Self-reported pregnancy complications consisted of anaemia, hypertension, diabetes, intrahepatic cholestasis, and so on.

\subsection{Statistical Analysis}

Maternal dietary pattern during pregnancy was obtained by the reduced rank regression (RRR) method using the PLS procedure in SAS [17]. Before conducting this analysis, 107 food items were categorised into 21 food groups according to their similarities in nutrient content and culinary usage (Table S1). The predictor variables were the daily intakes of 21 food groups in grams, while the response variables were the daily intakes of thiamin (vitamin B1), riboflavin (vitamin B2), niacin (vitamin B3), vitamin B6, folate, and vitamin B12 in grams. The number of RRR factors derived was equal to the number of response variables. Considering that the first RRR factor explained the highest proportion of the variation in response variables, only this factor was considered as the dietary pattern of interest $[18,38]$. Food groups with an absolute factor loading $\geq 0.2$ were utilised to char- 
acterise the dietary pattern. The dietary pattern score of each participant was outputted by summing up the intakes of each food group and multiplying with the corresponding factor loadings and categorised into quartiles. Pearson's correlation coefficients and 95\% CIs between the dietary pattern score and each response variable were calculated.

Binary and continuous variables were presented as $n(\%)$ and means \pm SDs, respectively. Linear trends across the quartiles of dietary pattern score were estimated using Cochran-Armitage tests for maternal characteristics and univariate linear regression models for nutrient intakes. Given the significant within-group homogeneity for most birth outcomes at the county level, the generalised linear mixed model with a random intercept at the county level was employed to estimate the associations of maternal dietary pattern score with birth outcomes. The dietary pattern score was analysed both as a continuous variable (per 1-SD increase) and a categorised variable (the lowest quartile as the reference). To assess the robustness of these associations, a total of three models were constructed in sequence. Model 1 included no covariates. Model 2 included socio-demographic characteristics. Model 3 included all covariates in Model 2 plus health-related behaviours. The link functions of continuous and binary dependent variables were "identity" and "logit", separately. Linear trends for birth outcomes across the quartiles of dietary pattern score were assessed by putting the quartile number into models as an ordinal variable. Since multivitamins usually contain B vitamins, stratified analyses by folic acid and multivitamin supplementation were performed to test whether these supplements modified the associations of dietary pattern score with birth outcomes. Potential effect modifications were examined by including interaction terms in the fully adjusted models.

All statistical analyses were performed using SAS software (version 9.4; SAS Institute Inc., Cary, CA, USA). A two-sided $p<0.05$ was considered statistically significant.

\section{Results}

\subsection{Dietary Pattern}

A total of six RRR factors were identified. The first RRR factor explained $42.27 \%$ of thiamin, $68.34 \%$ of riboflavin, $20.92 \%$ of niacin, $59.00 \%$ of vitamin $\mathrm{B} 6,56.16 \%$ of folate, $41.50 \%$ of vitamin B12, and $48.03 \%$ of the total variation of six B vitamins. The subsequent five RRR factors explained only $12.73 \%, 4.71 \%, 1.67 \%, 1.45 \%$, and $1.01 \%$ of the total variation, separately. Therefore, only the first RRR factor was regarded as the dietary pattern of interest. Since the dietary pattern score was moderately to highly positively correlated with the intakes of six B vitamins (Table 1), this pattern was named the high B-vitamin dietary pattern.

Table 1. Associations between the dietary pattern score derived by reduced rank regression and B vitamins intakes.

\begin{tabular}{ccc}
\hline & $\boldsymbol{r}(\mathbf{9 5} \% \mathbf{C I})$ & $\boldsymbol{p}$ \\
\hline Thiamin & $0.677(0.664,0.689)$ & $<0.001$ \\
Riboflavin & $0.866(0.860,0.872)$ & $<0.001$ \\
Niacin & $0.578(0.562,0.593)$ & $<0.001$ \\
Vitamin B6 & $0.809(0.801,0.817)$ & $<0.001$ \\
Folate & $0.841(0.834,0.847)$ & $<0.001$ \\
Vitamin B12 & $0.686(0.674,0.698)$ & $<0.001$ \\
\hline
\end{tabular}

The factor loading values of the food groups in the high B-vitamin dietary pattern are shown in Figure 1. The dietary pattern was characterised by high intakes of organ meat; fungi and algae; green vegetables; other vegetables; meat and poultry; legumes; fish, shrimps, and crabs; and low intakes of oils and cereals. As displayed in Table S2, the mean intakes of the selected nutrient varied across quartiles of the high B-vitamin dietary pattern score. On the whole, with the increasing quartiles, women tended to have lower intakes of carbohydrates, the percentage of energy from carbohydrates, potassium, and sodium 
but higher intakes of protein, the percentage of energy from protein, and most vitamins and minerals.

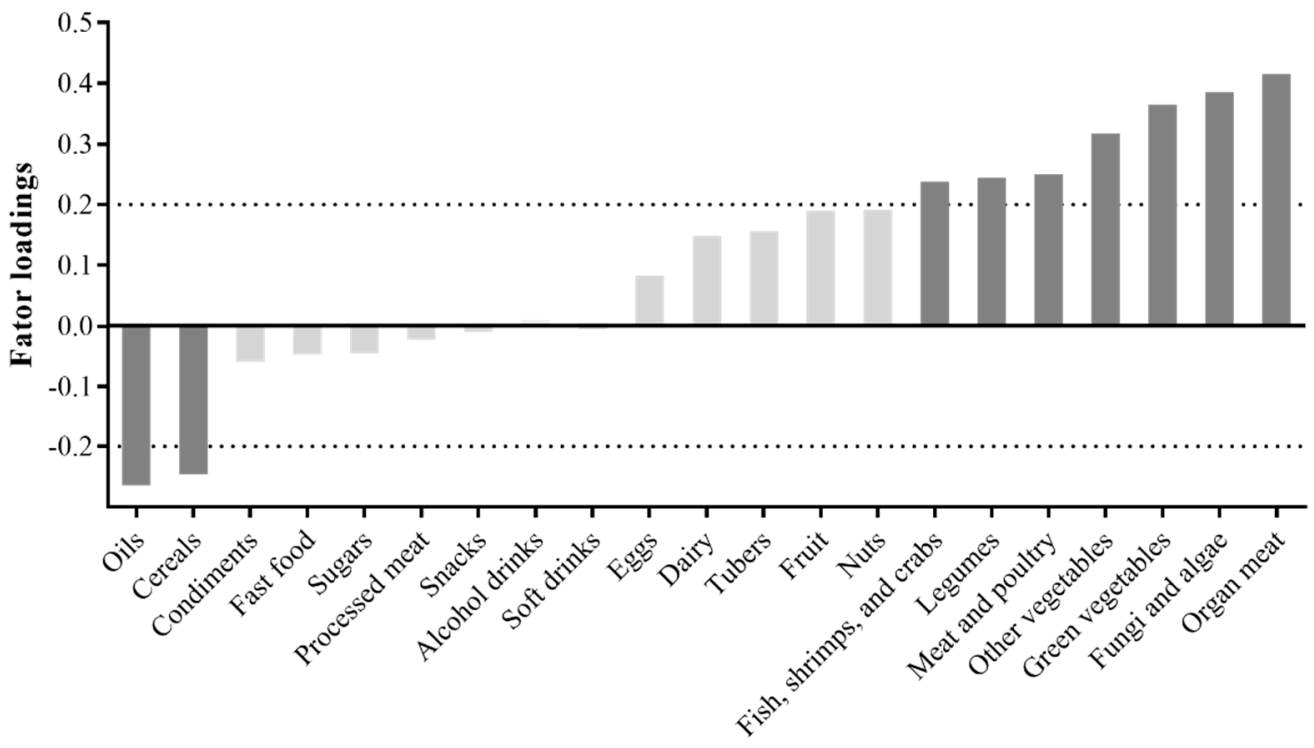

Food groups

Figure 1. Factor loading of the food groups in the high B-vitamin dietary pattern score. Dark grey indicates absolute factor loading value $\geq 0.2$.

\subsection{Participants Characteristics}

Maternal socio-demographic characteristics and health-related behaviours differed by quartiles of the high B-vitamin dietary pattern score (Table 2). Women in the highest quartile were more likely to be primiparous, give birth at the ages of 25-29 years, have more than junior school education, drink alcohol, and take iron, calcium, and folic acid supplements. By contrast, women in the highest quartile were less likely to be farmers, be poorer, be exposed to passive smoking, live in rural areas, have pregnancy complications, and use medication.

Table 2. Maternal characteristics according to quartiles of the high B-vitamin dietary pattern score.

\begin{tabular}{|c|c|c|c|c|c|c|}
\hline \multirow[t]{2}{*}{ Characteristics } & \multirow[t]{2}{*}{ Total } & \multicolumn{4}{|c|}{ Quartile } & \multirow[t]{2}{*}{$p_{\text {trend }}{ }^{\mathrm{a}}$} \\
\hline & & Q1 & Q2 & Q3 & Q4 & \\
\hline$N$ & 7347 & 1837 & 1837 & 1837 & 1836 & \\
\hline \multicolumn{7}{|l|}{ Socio-demographic characteristics } \\
\hline Geographic area (central Shaanxi) & $3993(54.35)$ & $994(54.11)$ & $1002(54.55)$ & $956(52.04)$ & $1041(56.70)$ & 0.311 \\
\hline Residence (rural) & $5612(76.38)$ & $1560(84.92)$ & $1530(83.29)$ & 1395 (75.94) & $1127(61.38)$ & $<0.001$ \\
\hline Age at delivery (25-29 years) & $2721(37.35)$ & $650(35.66)$ & $664(36.50)$ & $667(36.51)$ & 740 (40.73) & 0.003 \\
\hline Education (more than junior school) & $2726(37.23)$ & $561(30.62)$ & $577(31.46)$ & $671(36.67)$ & $917(50.19)$ & $<0.001$ \\
\hline Occupation (farmer) & $5266(72.21)$ & $1406(77.00)$ & 1404 (76.93) & 1332 (73.11) & $1124(61.76)$ & $<0.001$ \\
\hline Household wealth index (poor) & $2474(33.67)$ & $684(37.23)$ & $686(37.34)$ & $592(32.23)$ & $512(27.89)$ & $<0.001$ \\
\hline Parity (primiparous) & $4570(62.22)$ & $1088(59.23)$ & $1080(58.82)$ & $1141(62.15)$ & $1261(68.68)$ & $<0.001$ \\
\hline \multicolumn{7}{|l|}{ Health-related behaviours } \\
\hline Passive smoking & $1639(22.37)$ & $463(25.23)$ & $430(23.50)$ & $420(22.93)$ & $326(17.81)$ & $<0.001$ \\
\hline Alcohol consumption & $97(1.32)$ & $23(1.25)$ & $15(0.82)$ & $24(1.31)$ & $35(1.91)$ & 0.040 \\
\hline Pregnancy complications & $1521(20.71)$ & $394(21.46)$ & $405(22.07)$ & $385(20.96)$ & 337 (18.37) & 0.014 \\
\hline Medication use & $1394(19.02)$ & $380(20.71)$ & 359 (19.61) & 339 (18.49) & $316(17.26)$ & 0.005 \\
\hline Folic acid supplementation & $5430(74.25)$ & $1340(73.18)$ & $1333(72.96)$ & $1328(72.57)$ & $1429(78.30)$ & 0.001 \\
\hline Calcium supplementation & $4796(65.76)$ & $1172(64.18)$ & $1194(65.46)$ & $1170(63.93)$ & $1260(69.50)$ & 0.004 \\
\hline Iron supplementation & $727(9.93)$ & $156(8.51)$ & $171(9.36)$ & $196(10.72)$ & 204 (11.15) & 0.003 \\
\hline Multivitamin supplementation & $647(8.85)$ & $130(7.10)$ & $152(8.33)$ & $165(9.03)$ & $200(10.93)$ & $<0.001$ \\
\hline
\end{tabular}

Values are $n(\%)$. ${ }^{a}$ Obtained from Cochran-Armitage trend tests.

\subsection{Dietary Pattern and Birth Outcomes}

Associations of the high B-vitamin dietary pattern score with birth outcomes are presented in Tables 3 and 4. After adjustment for socio-demographic characteristics and 
health-related behaviours, the 1-SD increase in the high B-vitamin dietary pattern score was associated with $16.4 \mathrm{~g}$ (95\% CI: 5.4, $27.4 \mathrm{~g}$ ) higher birth weight, 0.040 (95\% CI: 0.014, 0.065 ) higher birth weight $Z$ score, and $12.1 \%$ (OR: 0.879; 95\% CI: 0.809, 0.955) lower risk of SGA. Compared with women in the lowest quartile, those in the highest quartile had newborns with a $44.5 \mathrm{~g}\left(95 \% \mathrm{CI}: 13.8,75.2 \mathrm{~g}\right.$; $\left.p_{\text {trend }}=0.012\right)$ higher birth weight, $0.101(95 \%$ CI: $\left.0.029,0.172 ; p_{\text {trend }}=0.012\right)$ higher birth weight $Z$ score, and $27.2 \%(\mathrm{OR}: 0.728 ; 95 \% \mathrm{CI}$ : $0.582,0.910 ; p_{\text {trend }}=0.026$ ) lower risk of SGA.

Table 3. Associations between the high B-vitamin dietary pattern score and continuous birth outcomes ${ }^{\text {a }}$.

\begin{tabular}{|c|c|c|c|c|c|c|}
\hline & \multirow[t]{2}{*}{ Continuous $^{\mathrm{b}}$} & \multicolumn{4}{|c|}{ Quartile } & \multirow[t]{2}{*}{$p_{\text {trend }}$} \\
\hline & & Q1 & Q2 & Q3 & Q4 & \\
\hline \multicolumn{7}{|c|}{ Birth weight, $\mathrm{g}^{\mathrm{d}}$} \\
\hline Mean \pm SD & $3270.2 \pm 448.1$ & $3244.4 \pm 452.0$ & $3269.5 \pm 451.5$ & $3259.5 \pm 451.0$ & $3307.4 \pm 435.6$ & \\
\hline Model $1^{\mathrm{e}}$ & $18.1(7.5,28.7)$ & Ref & $26.2(-2.8,55.3)$ & $12.5(-16.8,41.8)$ & $52.3(22.5,82.2)$ & 0.002 \\
\hline Model $2^{\mathrm{f}}$ & $16.3(5.5,27.1)$ & Ref & $25.3(-3.9,54.6)$ & $8.3(-21.2,37.8)$ & $45.4(15.0,75.7)$ & 0.010 \\
\hline Model $3 \mathrm{~g}$ & $16.4(5.4,27.4)$ & Ref & $21.9(-7.6,51.5)$ & $6.4(-23.4,36.1)$ & $44.5(13.8,75.2)$ & 0.012 \\
\hline \multicolumn{7}{|c|}{$\begin{array}{l}\text { Gestational age at } \\
\text { birth, weeks }\end{array}$} \\
\hline Mean \pm SD & $39.54 \pm 1.50$ & $39.55 \pm 1.32$ & $39.55 \pm 1.38$ & $39.55 \pm 1.60$ & $39.53 \pm 1.69$ & \\
\hline Model $1^{\mathrm{e}}$ & $0.002(-0.033,0.038)$ & Ref & $-0.020(-0.116,0.077)$ & $-0.003(-0.101,0.094)$ & $0.010(-0.090,0.109)$ & 0.785 \\
\hline Model $2^{\mathrm{f}}$ & $0.002(-0.034,0.038)$ & Ref & $-0.011(-0.108,0.086)$ & $-0.002(-0.100,0.096)$ & $0.012(-0.089,0.114)$ & 0.777 \\
\hline Model $3 \mathrm{~g}$ & $-0.001(-0.037,0.037)$ & Ref & $-0.006(-0.105,0.092)$ & $-0.004(-0.104,0.095)$ & $0.008(-0.095,0.111)$ & 0.870 \\
\hline \multicolumn{7}{|c|}{ Birth weight $Z$ score } \\
\hline Mean \pm SD & $-0.03 \pm 1.04$ & $-0.09 \pm 1.05$ & $-0.03 \pm 1.04$ & $-0.05 \pm 1.07$ & $0.07 \pm 1.01$ & \\
\hline Model 1 e & $0.042(0.017,0.067)$ & Ref & $0.073(0.005,0.140)$ & $0.032(-0.036,0.100)$ & $0.116(0.047,0.186)$ & 0.002 \\
\hline Model $2^{\mathrm{f}}$ & $0.039(0.014,0.064)$ & Ref & $0.069(0.002,0.137)$ & $0.023(-0.045,0.091)$ & $0.101(0.030,0.171)$ & 0.010 \\
\hline Model $3 \mathrm{~g}$ & $0.040(0.014,0.065)$ & Ref & $0.059(-0.009,0.128)$ & $0.019(-0.050,0.088)$ & $0.101(0.029,0.172)$ & 0.012 \\
\hline
\end{tabular}

Ref, reference. ${ }^{a}$ Two-level generalised linear mixed models were used to estimate mean differences and $95 \%$ CIs. ${ }^{b}$ Per 1-SD increase in the high B-vitamin dietary pattern score. ${ }^{c}$ Obtained using the median value of each dietary pattern quartile as a continuous variable in the regression models. ${ }^{\mathrm{d}}$ Precise to $10 \mathrm{~g} .{ }^{\mathrm{e}}$ Unadjusted. ${ }^{\mathrm{f}}$ Adjusted for socio-demographic characteristics, including geographic area, residence, age at delivery, education, occupation, household wealth index and parity. g Adjusted for all variables in model 2 plus health-related behaviours, including passive smoking, alcohol consumption, pregnancy complications, medication use, as well as iron, calcium, folic acid, and multivitamin supplementation.

Table 4. Associations between the high B-vitamin dietary pattern score and dichotomous birth outcomes ${ }^{\text {a }}$.

\begin{tabular}{|c|c|c|c|c|c|c|}
\hline & \multirow[t]{2}{*}{ Continuous ${ }^{b}$} & \multicolumn{4}{|c|}{ Quartile } & \multirow[t]{2}{*}{$p_{\text {trend }}$} \\
\hline & & Q1 & Q2 & Q3 & Q4 & \\
\hline \multicolumn{7}{|l|}{ LBW } \\
\hline$n(\%)$ & $226(3.10)$ & $58(3.20)$ & $59(3.24)$ & $64(3.51)$ & $45(2.47)$ & \\
\hline Model $1 \mathrm{~d}$ & $0.901(0.782,1.039)$ & Ref & $1.018(0.704,1.473)$ & $1.111(0.773,1.598)$ & $0.771(0.518,1.149)$ & 0.256 \\
\hline Model 2 e & $0.909(0.786,1.052)$ & Ref & $1.004(0.693,1.455)$ & $1.129(0.784,1.626)$ & $0.796(0.529,1.196)$ & 0.375 \\
\hline Model $3^{f}$ & $0.923(0.797,1.069)$ & Ref & $1.009(0.695,1.465)$ & $1.125(0.780,1.623)$ & $0.833(0.553,1.255)$ & 0.501 \\
\hline \multicolumn{7}{|l|}{ Preterm } \\
\hline$n(\%)$ & $227(3.09)$ & $60(3.27)$ & $64(3.49)$ & $57(3.11)$ & $46(2.51)$ & \\
\hline Model 1 d & $0.940(0.817,1.081)$ & Ref & $1.077(0.751,1.544)$ & $0.925(0.637,1.344)$ & $0.718(0.481,1.072)$ & 0.077 \\
\hline Model 2 e & $0.940(0.815,1.086)$ & Ref & $1.065(0.739,1.534)$ & $0.895(0.613,1.307)$ & $0.699(0.464,1.053)$ & 0.062 \\
\hline Model $3^{f}$ & $0.944(0.817,1.092)$ & Ref & $1.042(0.721,1.506)$ & $0.885(0.604,1.295)$ & $0.701(0.463,1.061)$ & 0.070 \\
\hline \multicolumn{7}{|l|}{ SGA } \\
\hline$n(\%)$ & $843(11.54)$ & $244(13.33)$ & $205(11.22)$ & $234(12.81)$ & $160(8.79)$ & \\
\hline Model 1 d & $0.852(0.786,0.924)$ & Ref & $0.818(0.669,0.999)$ & $0.977(0.803,1.189)$ & $0.660(0.532,0.821)$ & 0.001 \\
\hline Model $2 \mathrm{e}$ & $0.874(0.805,0.948)$ & Ref & $0.811(0.662,0.992)$ & $0.988(0.810,1.204)$ & $0.705(0.566,0.879)$ & 0.011 \\
\hline Model $3^{f}$ & $0.879(0.809,0.955)$ & Ref & $0.831(0.678,1.020)$ & $1.016(0.832,1.241)$ & $0.728(0.582,0.910)$ & 0.026 \\
\hline
\end{tabular}

LBW, low birth weight; Ref, reference; SGA, small-for-gestational-age. ${ }^{a}$ Two-level generalised linear mixed models were used to estimate ORs and $95 \%$ CIs. ${ }^{\text {b }}$ Per 1-SD increase in the high B-vitamin dietary pattern score. ${ }^{c}$ Obtained using the median value of each dietary pattern quartile as a continuous variable in the regression models. ${ }^{\mathrm{d}}$ Unadjusted. ${ }^{\mathrm{e}}$ Adjusted for socio-demographic characteristics, including geographic area, residence, age at delivery, education, occupation, household wealth index and parity. ${ }^{\mathrm{f}}$ Adjusted for all variables in model 2 plus health-related behaviours, including passive smoking, alcohol consumption, pregnancy complications, medication use, as well as iron, calcium, folic acid, and multivitamin supplementation. 
Stratified analyses according to folic acid and multivitamin supplementation are displayed in Figures S2-S4. A marginally significant interaction was observed between multivitamin supplementation and dietary pattern score on SGA risk $\left(P_{\text {interaction }}=0.056\right)$. Among the women who did not use multivitamins, per 1-SD increase in the high B-vitamin dietary pattern score was related to $14.2 \%$ (OR: $0.858 ; 95 \%$ CI: $0.786,0.937$ ) lower risk of SGA. In contrast, the association was not significant among users $(p>0.05)$ (Figure S4).

\section{Discussion}

This population-based study conducted in northwest China identified a high B-vitamin dietary pattern, which was characterised by high intakes of animal foods, vegetables, fungi and algae, legumes, and low intakes of oils and cereals. A higher score for the dietary pattern corresponded to higher birth weight and Z score as well as a lower risk of SGA.

To our knowledge, only one other study examined the associations of maternal dietary patterns characterised by B vitamins with neonatal outcomes [39]. In a mother-child cohort in France, 1638 pregnant women before 24 weeks of gestation were recruited to report their diet in the year before pregnancy retrospectively. As compared with our study, the authors regarded one-carbon metabolism nutrients including riboflavin, vitamin B6, folate, vitamin B12, betaine, choline, and methionine as response variables. They also derived a dietary pattern rich in B vitamins, which was loaded positively with low-fat milk, meat, liver, fish, eggs, cereals, mixed vegetables, chicory, leek and cabbage, and broccoli but loaded negatively with snacks and confectionery and sugar-sweetened beverages [39]. The similarity between the dietary patterns in their study and those of ours was that food groups were diverse and balanced. The difference was that our pattern had low intakes of cereals, which may be attributed to the variation in response variables and composition of cereals. The cereals we classified included wheat and rice products, whereas the cereals in their study referred to breakfast cereals and cereal bar fruits. In general, as the bran and germ are removed, commercially available cereals contain small amounts of B vitamins (thiamin, riboflavin, niacin, and folate). Given the protective effects of folic acid on neural tube defects, many countries have created legislation to mandate the fortification of industrially milled cereals with folic acid [40]. However, such projects have not yet been implemented both in China and France. The authors indicated that, when controlling for potential confounders, adherence to the high B-vitamin dietary pattern before pregnancy was not related to birth weight, gestational age, and the risk of SGA [39]. However, in our study, adherence to this dietary pattern during pregnancy was positively associated with birth weight and inversely associated with the risk of SGA. These results may be partly explained by the wide gap in nutritional status between the two populations. The intakes of riboflavin, vitamin B6, folate, and vitamin B12 in our population were approximately $15-65 \%$ lower compared with theirs. The benefits of micronutrients were more likely to be observed in malnourished populations. In addition, the timing of interest in the two studies was different. In terms of intrauterine growth, the role of maternal nutrition during pregnancy was far more prominent than that of pre-pregnancy nutrition.

In the present study, it seemed that the high B-vitamins dietary pattern exerted protective effects against SGA only among pregnant women who did not take multivitamins. A plausible explanation for this finding was that multivitamins provided adequate B vitamins to meet the needs of fetal growth. Owing to the variation in the brands of multivitamins, the content of B vitamins in supplements cannot be directly compared with the dietary pattern. Based on a double-blind randomised controlled trial in rural Shaanxi Province, we previously reported that antenatal multiple micronutrients with a recommended allowance of $B$ vitamins resulted in a $44 \mathrm{~g}$ increase in birth weight and a 0.19 week increase in gestational age in comparison with folic acid alone [41]. Overall, these studies suggested that prenatal nutrition intervention focusing on B vitamins is probably an effective approach to improve birth outcomes in northwest China.

This study found that neither the continuous nor binary variable of gestational age was relevant to the high B-vitamin dietary pattern. On the contrary, birth weight-related 
outcomes, including crude birth weight, gestational age- and sex-specific birth weight, and SGA, were all associated with the high B-vitamin dietary pattern. These results suggest that birth weight rather than length of gestation is susceptible to the dietary pattern. Birth weight is the most widely used anthropometric indicator for newborns, in which an increase indicates the promotion of growth and development [42]. In 2012, an estimated 874,000 babies were born SGA in China, with 15,100 attributable neonatal deaths [43]. Babies born SGA are also reported to have a higher risk of delayed neurodevelopment and of being underweight in early adolescence [44,45]. The finding that adherence to the high B-vitamin dietary pattern was associated with a moderate reduction in SGA risk provides one possibility for achieving Sustainable Development Goal 3 (ensuring healthy lives and promoting well-being at all ages). Well-designed longitudinal studies are warranted to validate our findings and to explore the long-term effects of maternal dietary patterns.

Mechanisms that relate antenatal B vitamins and birth weight are still unclear. Almost all $\mathrm{B}$ vitamins are involved in one-carbon metabolism and related pathways. Folate is the main carrier of one-carbon units, while riboflavin, vitamin B6, and vitamin B12 act as essential cofactors or precursors of key enzymes [46]. Importantly, one-carbon metabolism plays a role in cellular processes such as biosynthesis, amino acid homeostasis, epigenetic regulation, and redox defence [47]. A growing body of clinical trials have shown that prenatal B vitamins can reduce homocysteine concentrations or alter DNA methylation patterns among newborns [48-50], but whether these changes further interfere with birth weight remains to be understood.

It is noteworthy that although our dietary pattern was driven by the variability of B vitamins, the highest quartile was accompanied by the highest intakes of other nutrients, such as protein, vitamin A, vitamin C, calcium, and zinc. We cannot exclude the potential effects of these nutrients on birth weight. Rather than single out individual nutrients that could account for the benefits of the dietary pattern studied, it may be more realistic to explore the synergy of multiple nutrients or food groups.

The present study has some strengths. First, because of the stratified multistage random sampling method, our findings can be generalised to the whole Shaanxi Province. Second, in contrast with previous studies that investigated the role of individual B vitamins in neonatal outcomes, our study explored the beneficial effect of the B vitamins-related dietary pattern using the RRR method, which provides a better foundation for the development of dietary recommendations for pregnant women. Nevertheless, several limitations should be noted. First, temporality and causality cannot be demonstrated from this crosssectional study. Second, residual confounding cannot be ruled out due to unmeasured or unknown socio-demographic or health-related factors. Third, women were asked to recall pregnancy characteristics within 0-12 months after delivery in our study. Although many studies have shown that pregnancy is a major event during which many features can be recalled well even after years [51,52], the accuracy of our data remains to be validated. Finally, given the convenience and low cost, we evaluated the average dietary intake throughout pregnancy, which was likely to underestimate the importance of maternal diets during a certain stage. Indeed, accumulating evidence indicates that fetal growth is most affected by micronutrient deficiencies at the very earliest embryonic stages $[53,54]$. Prospective cohort studies that collect maternal dietary intakes over multiple time points from the periconceptional period onwards are required.

\section{Conclusions}

In the cross-sectional study in northwest China, we derived a maternal dietary pattern that was rich in B vitamins using the RRR method and found that greater adherence to this dietary pattern during pregnancy was related to higher birth weight and a lower risk of SGA. Based on these findings, obstetricians should pay more attention to the B-vitamin status of pregnant women and advise women to increase the proportion of animal foods, vegetables, fungi and algae, and legumes in their diets to prevent adverse birth outcomes. 
Supplementary Materials: The following supporting information can be downloaded at: https: / / www.mdpi.com/article/10.3390/nu14030600/s1, Table S1: Foods items within each food group; Table S2: Selected nutrient intakes according to quartiles of the high B-vitamin dietary pattern score; Figure S1: Flow chart of the selection of study participants; Figure S2: Associations of 1-SD increase in the high B-vitamin dietary pattern score with birth weight stratified by the use of B vitaminscontaining supplements; Figure S3: Associations of 1-SD increase in the high B-vitamin dietary pattern score with birth weight $Z$ score stratified by the use of B vitamins-containing supplements; Figure S4: Associations of 1-SD increase in the high B-vitamin dietary pattern score with SGA stratified by the use of B vitamins-containing supplements.

Author Contributions: L.Z., S.D. and H.Y. conceived and designed the cross-sectional study; Y.K., P.Q., B.M., Z.Z., L.P., L.Z. and S.D. conducted the study; S.L., D.L., L.H., Y.Z., F.C. and D.W. performed the statistical analysis; L.Z. and S.D. wrote the manuscript. All authors have read and agreed to the published version of the manuscript.

Funding: This research was funded by the National Key Research and Development Program of China (grant numbers: 2017YFC0907200 and 2017YFC0907201), the National Natural Science Foundation of China (grant numbers: 81230016 and 72174167), and the Project of birth defect control and prevention in Shaanxi (grant number: Sxwsjswzfcght2016-013).

Institutional Review Board Statement: The cross-sectional study was conducted following the Declaration of Helsinki, and approved by the ethics committee of the Xi'an Jiaotong University Health Science Center (No. 2012008).

Informed Consent Statement: Informed consent was obtained from all subjects involved in the study.

Acknowledgments: The authors are grateful to all participants and investigators in the present study.

Conflicts of Interest: The authors declare no conflict of interest. The funders had no role in the design of the study; in the collection, analyses, or interpretation of data; in the writing of the manuscript, or in the decision to publish the results.

\section{References}

1. Katz, J.; Lee, A.C.; Kozuki, N.; Lawn, J.E.; Cousens, S.; Blencowe, H.; Ezzati, M.; Bhutta, Z.A.; Marchant, T.; Willey, B.A.; et al. Mortality risk in preterm and small-for-gestational-age infants in low-income and middle-income countries: A pooled country analysis. Lancet 2013, 382, 417-425. [CrossRef]

2. Jin, J. JAMA patient page. Babies with low birth weight. JAMA 2015, 313, 432. [CrossRef]

3. Barker, D.J. Fetal origins of coronary heart disease. BMJ 1995, 311, 171-174. [CrossRef]

4. Barker, D.J.; Osmond, C. Infant mortality, childhood nutrition, and ischaemic heart disease in England and Wales. Lancet 1986, 1, 1077-1081. [CrossRef]

5. Osmond, C.; Barker, D.J.; Winter, P.D.; Fall, C.H.; Simmonds, S.J. Early growth and death from cardiovascular disease in women. BMJ 1993, 307, 1519-1524. [CrossRef]

6. Department of Maternal and Child Health. China Maternal and Child Health Development Report (2019). Available online: http:/ / www.nhc.gov.cn/fys/s7901/201905/bbd8e2134a7e47958c5c9ef032e1dfa2.shtml (accessed on 23 December 2021).

7. Chawanpaiboon, S.; Vogel, J.P.; Moller, A.-B.; Lumbiganon, P.; Petzold, M.; Hogan, D.; Landoulsi, S.; Jampathong, N.; Kongwattanakul, K.; Laopaiboon, M.; et al. Global, regional, and national estimates of levels of preterm birth in 2014: A systematic review and modelling analysis. Lancet Glob. Health 2019, 7, e37-e46. [CrossRef]

8. $\quad$ Blencowe, H.; Krasevec, J.; de Onis, M.; Black, R.E.; An, X.; Stevens, G.A.; Borghi, E.; Hayashi, C.; Estevez, D.; Cegolon, L.; et al. National, regional, and worldwide estimates of low birthweight in 2015, with trends from 2000: A systematic analysis. Lancet Glob. Health 2019, 7, e849-e860. [CrossRef]

9. Lee, A.C.C.; Katz, J.; Blencowe, H.; Cousens, S.; Kozuki, N.; Vogel, J.P.; Adair, L.; Baqui, A.H.; Bhutta, Z.A.; Caulfield, L.E.; et al. National and regional estimates of term and preterm babies born small for gestational age in 138 low-income and middle-income countries in 2010. Lancet Glob. Health 2013, 1, e26-e36. [CrossRef]

10. Abu-Saad, K.; Fraser, D. Maternal nutrition and birth outcomes. Epidemiol. Rev. 2010, 32, 5-25. [CrossRef]

11. Lyon, P.; Strippoli, V.; Fang, B.; Cimmino, L. B Vitamins and one-carbon metabolism: Implications in human health and disease. Nutrients 2020, 12, 2867. [CrossRef]

12. Salcedo-Bellido, I.; Martínez-Galiano, J.M.; Olmedo-Requena, R.; Mozas-Moreno, J.; Bueno-Cavanillas, A.; Jimenez-Moleon, J.J.; Delgado-Rodríguez, M. Association between vitamin intake during pregnancy and risk of small for gestational age. Nutrients 2017, 9, 1277. [CrossRef]

13. Krapels, I.P.; van Rooij, I.A.; Ocké, M.C.; van Cleef, B.A.; Kuijpers-Jagtman, A.M.; Steegers-Theunissen, R.P. Maternal dietary B vitamin intake, other than folate, and the association with orofacial cleft in the offspring. Eur. J. Nutr. 2004, 43, 7-14. [CrossRef] 
14. Verkleij-Hagoort, A.C.; de Vries, J.H.M.; Ursem, N.T.C.; de Jonge, R.; Hop, W.C.J.; Steegers-Theunissen, R.P.M. Dietary intake of B-vitamins in mothers born a child with a congenital heart defect. Eur. J. Nutr. 2006, 45, 478-486. [CrossRef]

15. Hu, F.B. Dietary pattern analysis: A new direction in nutritional epidemiology. Curr. Opin. Lipidol. 2002, 13, 3-9. [CrossRef]

16. Nakayama, A.T.; Lutz, L.J.; Hruby, A.; Karl, J.P.; McClung, J.P.; Gaffney-Stomberg, E. A dietary pattern rich in calcium, potassium, and protein is associated with tibia bone mineral content and strength in young adults entering initial military training. Am. J. Clin. Nutr. 2019, 109, 186-196. [CrossRef]

17. Hoffmann, K.; Schulze, M.B.; Schienkiewitz, A.; Nöthlings, U.; Boeing, H. Application of a new statistical method to derive dietary patterns in nutritional epidemiology. Am. J. Epidemiol. 2004, 159, 935-944. [CrossRef]

18. Hoffmann, K.; Zyriax, B.C.; Boeing, H.; Windler, E. A dietary pattern derived to explain biomarker variation is strongly associated with the risk of coronary artery disease. Am. J. Clin. Nutr. 2004, 80, 633-640. [CrossRef]

19. Li, S.; Liu, D.; Zhang, R.; Lei, F.; Liu, X.; Cheng, Y.; Li, C.; Xiao, M.; Guo, L.; Li, M.; et al. The association of maternal dietary folate intake and folic acid supplementation with small-for-gestational-age births: A cross-sectional study in Northwest China. Br. J. Nutr. 2019, 122, 459-467. [CrossRef]

20. Li, S.; Lei, F.; Zhang, R.; Liu, D.; Qu, P.; Cheng, Y.; Liu, X.; Chen, F.; Dang, S.; Yan, H. Socioeconomic disparity in the diet quality of pregnant women in Northwest China. Asia Pac. J. Clin. Nutr. 2019, 28, 330-340. [CrossRef]

21. Meltzer, H.M.; Brantsaeter, A.L.; Ydersbond, T.A.; Alexander, J.; Haugen, M. Methodological challenges when monitoring the diet of pregnant women in a large study: Experiences from the Norwegian Mother and Child Cohort Study (MoBa). Matern. Child Nutr. 2008, 4, 14-27. [CrossRef]

22. Rifas-Shiman, S.L.; Rich-Edwards, J.W.; Willett, W.C.; Kleinman, K.P.; Oken, E.; Gillman, M.W. Changes in dietary intake from the first to the second trimester of pregnancy. Paediatr. Perinat. Epidemiol. 2006, 20, 35-42. [CrossRef] [PubMed]

23. Cucó, G.; Fernández-Ballart, J.; Sala, J.; Viladrich, C.; Iranzo, R.; Vila, J.; Arija, V. Dietary patterns and associated lifestyles in preconception, pregnancy and postpartum. Eur. J. Clin. Nutr. 2006, 60, 364-371. [CrossRef] [PubMed]

24. Crozier, S.R.; Robinson, S.M.; Godfrey, K.M.; Cooper, C.; Inskip, H.M. Women's dietary patterns change little from before to during pregnancy. J. Nutr. 2009, 139, 1956-1963. [CrossRef] [PubMed]

25. Cheng, Y.; Yan, H.; Dibley, M.J.; Shen, Y.; Li, Q.; Zeng, L. Validity and reproducibility of a semi-quantitative food frequency questionnaire for use among pregnant women in rural China. Asia Pac. J. Clin. Nutr. 2008, 17, 166-177.

26. Cheng, Y.; Zhou, X.; Zhang, X.; Zeng, L.; Yan, H. Food Frequency Questionnaire and Atlas; Shaanxi Science and Technology Press: Xi'an, China, 2009.

27. Institute of Nutrition and Food Safety; China Center for Disease Control and Prevention. China Food Composition Book 1, 6th ed.; Peking University Medical Press: Beijing, China, 2018.

28. Institute of Nutrition and Food Safety; China Center for Disease Control and Prevention. China Food Composition Book 2, 6th ed.; Peking University Medical Press: Beijing, China, 2019.

29. Willett, W. Nutritional Epidemiology, 3rd ed.; Oxford University Press: New York, NY, USA, 2013.

30. Villar, J.; Cheikh Ismail, L.; Victora, C.G.; Ohuma, E.O.; Bertino, E.; Altman, D.G.; Lambert, A.; Papageorghiou, A.T.; Carvalho, M.; Jaffer, Y.A.; et al. International standards for newborn weight, length, and head circumference by gestational age and sex: The Newborn Cross-Sectional Study of the INTERGROWTH-21st Project. Lancet 2014, 384, 857-868. [CrossRef]

31. WHO. ICD-11 for Mortality and Morbidity Statistics. Available online: https://icd.who.int/browse11/1-m/en\#/http://id.who. int/icd/entity/2041060050 (accessed on 23 December 2021).

32. WHO. Recommended definitions, terminology and format for statistical tables related to the perinatal period and use of a new certificate for cause of perinatal deaths. Acta Obstet. Gynecol. Scand. 1977, 56, 247-253.

33. de Onis, M.; Habicht, J.P. Anthropometric reference data for international use: Recommendations from a World Health Organization Expert Committee. Am. J. Clin. Nutr. 1996, 64, 650-658. [CrossRef]

34. Liu, X.; Lv, L.; Zhang, H.; Zhao, N.; Qiu, J.; He, X.; Zhou, M.; Xu, X.; Cui, H.; Liu, S.; et al. Folic acid supplementation, dietary folate intake and risk of preterm birth in China. Eur. J. Nutr. 2016, 55, 1411-1422. [CrossRef]

35. Timmermans, S.; Jaddoe, V.W.V.; Hofman, A.; Steegers-Theunissen, R.P.M.; Steegers, E.A.P. Periconception folic acid supplementation, fetal growth and the risks of low birth weight and preterm birth: The Generation R Study. Br. J. Nutr. 2009, 102, 777-785. [CrossRef]

36. Zheng, J.S.; Guan, Y.; Zhao, Y.; Zhao, W.; Tang, X.; Chen, H.; Xu, M.; Wu, L.; Zhu, S.; Liu, H.; et al. Pre-conceptional intake of folic acid supplements is inversely associated with risk of preterm birth and small-for-gestational-age birth: A prospective cohort study. Br. J. Nutr. 2016, 115, 509-516. [CrossRef]

37. Pei, L.; Kang, Y.; Zhao, Y.; Cheng, Y.; Yan, H. Changes in socioeconomic inequality of low birth weight and macrosomia in Shaanxi province of Northwest China, 2010-2013: A cross-sectional study. Medicine 2016, 95, e2471. [CrossRef] [PubMed]

38. Maddock, J.; Ambrosini, G.L.; Griffin, J.L.; West, J.A.; Wong, A.; Hardy, R.; Ray, S. A dietary pattern derived using B-vitamins and its relationship with vascular markers over the life course. Clin. Nutr. 2019, 38, 1464-1473. [CrossRef] [PubMed]

39. Lecorguillé, M.; Lioret, S.; de Lauzon-Guillain, B.; de Gavelle, E.; Forhan, A.; Mariotti, F.; Charles, M.A.; Heude, B. Association between dietary intake of one-carbon metabolism nutrients in the year before pregnancy and birth anthropometry. Nutrients 2020, 12, 838. [CrossRef] [PubMed]

40. Crider, K.S.; Bailey, L.B.; Berry, R.J. Folic acid food fortification-its history, effect, concerns, and future directions. Nutrients 2011, 3 , 370-384. [CrossRef] [PubMed] 
41. Zeng, L.; Dibley, M.J.; Cheng, Y.; Dang, S.; Chang, S.; Kong, L.; Yan, H. Impact of micronutrient supplementation during pregnancy on birth weight, duration of gestation, and perinatal mortality in rural western China: Double blind cluster randomised controlled trial. BMJ 2008, 337, a2001. [CrossRef] [PubMed]

42. Ba-Saddik, I.A.; Al-Asbahi, T.O. Anthropometric measurements of singleton live full-term newborns in Aden, Yemen. Int. J. Pediatr. Adolesc. Med. 2020, 7, 121-126. [CrossRef]

43. Estimates of burden and consequences of infants born small for gestational age in low and middle income countries with INTERGROWTH-21st standard: Analysis of CHERG datasets. BMJ 2017, 358, j4229. [CrossRef]

44. Zhu, Z.; Perumal, N.; Fawzi, W.W.; Cheng, Y.; Elhoumed, M.; Qi, Q.; Wang, L.; Dibley, M.J.; Zeng, L.; Sudfeld, C.R. Postnatal stature does not largely mediate the relation between adverse birth outcomes and cognitive development in mid-childhood and early adolescence in rural western China. J. Nutr. 2022, 152, 302-309. [CrossRef] [PubMed]

45. Elhoumed, M.; Andegiorgish, A.K.; Qi, Q.; Gebremedhin, M.A.; Wang, L.; Uwimana, G.; Cheng, Y.; Zhu, Z.; Zeng, L. Patterns and determinants of the double burden of malnutrition among adolescents: A 14-year follow-up of a birth cohort in rural China. $J$. Pediatr. 2021. Online ahead of print. [CrossRef]

46. Anderson, O.S.; Sant, K.E.; Dolinoy, D.C. Nutrition and epigenetics: An interplay of dietary methyl donors, one-carbon metabolism and DNA methylation. J. Nutr. Biochem. 2012, 23, 853-859. [CrossRef]

47. Clare, C.E.; Brassington, A.H.; Kwong, W.Y.; Sinclair, K.D. One-carbon metabolism: Linking nutritional biochemistry to epigenetic programming of long-term development. Annu. Rev. Anim. Biosci. 2019, 7, 263-287. [CrossRef] [PubMed]

48. Li, S.; Mi, B.; Qu, P.; Liu, D.; Lei, F.; Wang, D.; Zeng, L.; Kang, Y.; Shen, Y.; Pei, L.; et al. Association of antenatal vitamin B complex supplementation with neonatal vitamin B12 status: Evidence from a cluster randomized controlled trial. Eur. J. Nutr. 2021, 60, 1031-1039. [CrossRef] [PubMed]

49. Caffrey, A.; Irwin, R.E.; McNulty, H.; Strain, J.J.; Lees-Murdock, D.J.; McNulty, B.A.; Ward, M.; Walsh, C.P.; Pentieva, K. Genespecific DNA methylation in newborns in response to folic acid supplementation during the second and third trimesters of pregnancy: Epigenetic analysis from a randomized controlled trial. Am. J. Clin. Nutr. 2018, 107, 566-575. [CrossRef] [PubMed]

50. Duggan, C.; Srinivasan, K.; Thomas, T.; Samuel, T.; Rajendran, R.; Muthayya, S.; Finkelstein, J.L.; Lukose, A.; Fawzi, W.; Allen, L.H.; et al. Vitamin B-12 supplementation during pregnancy and early lactation increases maternal, breast milk, and infant measures of vitamin B-12 status. J. Nutr. 2014, 144, 758-764. [CrossRef] [PubMed]

51. Bunin, G.R.; Gyllstrom, M.E.; Brown, J.E.; Kahn, E.B.; Kushi, L.H. Recall of diet during a past pregnancy. Am. J. Epidemiol. 2001, 154, 1136-1142. [CrossRef]

52. Bosco, J.L.; Tseng, M.; Spector, L.G.; Olshan, A.F.; Bunin, G.R. Reproducibility of reported nutrient intake and supplement use during a past pregnancy: A report from the Children's Oncology Group. Paediatr. Perinat. Epidemiol. 2010, 24, 93-101. [CrossRef]

53. Nafee, T.M.; Farrell, W.E.; Carroll, W.D.; Fryer, A.A.; Ismail, K.M. Epigenetic control of fetal gene expression. Bjog Int. J. Obstet. Gynaecol. 2008, 115, 158-168. [CrossRef]

54. Waterland, R.A.; Jirtle, R.L. Early nutrition, epigenetic changes at transposons and imprinted genes, and enhanced susceptibility to adult chronic diseases. Nutrition 2004, 20, 63-68. [CrossRef] 